\title{
The Origin of Magnetism in Mn-Doped $\mathrm{SrTiO}_{3}$
}

\author{
Matjaz Valant,* Taras Kolodiazhnyi, Iztok Arčon, Frederic Aguesse, Anna-Karin Axelsson, \\ and Neil M. Alford
}

An analysis of $\mathrm{Mn}$ substitution in $\mathrm{SrTiO}_{3}$ is performed in order to understand the origin of reported spin coupling in lightly $\mathrm{Mn}$-doped $\mathrm{SrTiO}_{3}$. The spin glass state magnetoelectrically coupled to the dipolar glass state has previously been reported for $\mathrm{SrTiO}_{3}$ substituted with only $2 \%$ of $\mathrm{Mn}$ on the B-site. An analysis of the substitution mechanism for A- and B-site doping shows a strong influence of processing conditions, such as processing temperature, oxygen partial pressure, and off-stoichiometry. The required conditions for a site-selective substitution are defined, which yield a single-phase and almost defect-free perovskite. Magnetic measurements show no magnetic anomalies resulting from spin coupling and only a simple paramagnetic behavior. Magnetic anomalies are observed only for the samples in which $\mathrm{Mn}$ is misplaced within the cation sublattice of the $\mathrm{SrTiO}_{3}$ perovskite. This occurs due to improper material processing, which causes initially unpredicted changes in the valence state of the $\mathrm{Mn}$ and results in the formation of structural defects and irregularities associated with segregation and nucleation of the magnetic species. Previously reported spin coupling in $\mathrm{Mn}$-doped $\mathrm{SrTiO}_{3}$ is not an intrinsic phenomenon and cannot be treated as a spin glass.

\section{Introduction}

Recently, oxide semiconductors doped with transition metals have been investigated for two major reasons; either in the quest for new dilute magnetic oxides (DMO) or for new multiferroic/magnetoelectric systems. Doping of oxide semiconductors with transition metals has often been considered as a way to induce a long-range magnetic ordering in a dielectric. Studies have been performed on simple oxides such as $\mathrm{ZnO}$, $\mathrm{Sn}_{2} \mathrm{O}, \mathrm{TiO}_{2}$ and $\mathrm{In}_{2} \mathrm{O}_{3},{ }^{[1-4]}$ as well as on more complex oxide compounds, such as $\mathrm{BaTiO}_{3}, \mathrm{KTaO}_{3}$ and $\mathrm{SrTiO}_{3}$, etc. ${ }^{[5]}$ The common characteristics of these studies are that they are mainly

Prof. M. Valant, Prof. I. Arčon

University of Nova Gorica

Vipavska 13, Nova Gorica, SI-5000, Slovenia

E-mail: matjaz.valant@ung.si

Dr. T. Kolodiazhnyi

National Institute for Materials Science

1-1 Namiki, Tsukuba, Ibaraki, 305-0044, Japan

Prof. I. Arčon

Institut Jozef Stefan

Jamova 39, Ljubljana, SI-1000, Slovenia

F. Aguesse, A.-K. Axelsson, N. M. Alford

Imperial College London

South Kensington Campus, London, SW7 2AZ, UK

DOI: 10.1002/adfm.201102482 quite controversial. The reported studies are often not comprehensive from all relevant perspectives. Very often, they have focused only on a fine characterization of the physical properties and physical modeling of the magnetic response, but have not reported detailed microstructural and crystallographic analyses. Only subsequent research has revealed problems related to the presence of extrinsic sources of magnetism, such as magnetic impurities, magnetic secondary phases, clustering and segregation of magnetic species, etc. A number of cases can be listed ( $\mathrm{Mn}: \mathrm{ZnO}$, $\mathrm{Mn}: \mathrm{KTaO}_{3}$, Co:ZnO, doped $\mathrm{In}_{2} \mathrm{O}_{3}$ ), for which researchers have recently expressed their concerns on the quality of the published results related to magnetism in lightly magnetic-doped oxide semiconductors, including potential DMO and magnetoelectric systems. ${ }^{[6-9]}$ The majority of the described magnetic phenomena in the bulk have so far been attributed to experimental artifacts and extrinsic factors. ${ }^{[10]}$ More likely, coherent, intrinsic, long-range spin ordering can be expected in nanoparticles and thin films, where it is associated with surface phenomena, especially at higher levels of oxygen vacancies at the surface/interface layers. ${ }^{[11,12]}$ There are models reported, but the exact atomistic mechanisms of the spin coupling in such systems have not yet been clearly confirmed. ${ }^{[13,14]}$

Here, we describe our research of yet another very intriguing system: $\mathrm{Mn}$-doped $\mathrm{SrTiO}_{3}$ has recently been widely investigated and it is claimed that it exhibits rather unusual and complex magnetic ordering below $40 \mathrm{~K}$, associated with magnetoelectric coupling. ${ }^{[15-17]}$ We have performed a comprehensive study on the Mn-incorporation as a function of the synthesis conditions in order to reveal the origin of the observed magnetic ordering. We show here that the current understanding of the magnetism in $\mathrm{Mn}$-doped $\mathrm{SrTiO}_{3}$ is deficient, mainly due to insufficient understanding of the substitutional chemistry in this system. We demonstrate that no magnetic order and, consequently, no magnetoelectric coupling exists in the bulk Mndoped $\mathrm{SrTiO}_{3}$, and that the observed magnetic anomalies result from extrinsic sources. However, first, we review the recent work and current understanding of the chemistry and magnetic properties of the $\mathrm{Mn}$-doped $\mathrm{SrTiO}_{3}$ material, which illustrates well the experimental difficulties and the controversy related to its characteristics.

Azzoni et al. ${ }^{[18]}$ investigated $\mathrm{Mn}: \mathrm{SrTiO}_{3}$ by electron paramagnetic resonance (EPR) and dc magnetic measurements to 
a doping level of up to $3 \%$. No information on the nominal substitution mechanism that was adopted during the synthesis of the powders was given in their work. The EPR data showed that, in $\mathrm{Mn}: \mathrm{SrTiO}_{3}$, different environments of the manganese ions in $\mathrm{Mn}^{4+}$ and $\mathrm{Mn}^{2+}$ oxidation states were present in different concentrations. Their results also showed an inhomogeneous Mn distribution over the sample. The dc magnetic data showed no distinct magnetic phase transition and no influence of a bias magnetic field on the magnetic curves. The spin interactions were identified to be only local antiferromagnetic interactions, as confirmed by negative values of the Weiss temperature. Nanosized inclusions of the secondary perovskite phase with a high Mn concentration were detected by X-ray diffraction (XRD) and by the EPR analysis; however, they could not be attributed to cubic $\mathrm{SrMnO}_{3}$ with an antiferromagnetic phase transition at $T_{\mathrm{N}}=240 \mathrm{~K} \cdot{ }^{[19]}$ Later work on Mn-implanted $\mathrm{SrTiO}_{3}$ has shown evidence of magnetic ordering. ${ }^{[5,20]}$ While $\mathrm{SrTiO}_{3}$ samples with 5 at\% Mn showed paramagnetic behavior, a clear signature of ferromagnetism near $300 \mathrm{~K}$ was noticed at 3 at\% Mn concentration. However, due to the nature of the ion implantation, for which penetration depth is only about $2000 \AA$, considerable structural disorder, oxygen vacancies, ion segregation and ion clustering were expected despite the post-annealing in $\mathrm{N}_{2}$. Further studies ${ }^{[21]}$ on the implanted systems were not consistent with the described results, which showed somewhat irreproducible characteristics of the ion implantation techniques. These systems cannot compare with chemically doped crystals that are close to their thermal phase equilibrium.

Recently, high-quality structural work has been performed on Mn-doped $\mathrm{SrTiO}_{3} \cdot{ }^{[22-24]}$ When discussing the structural mechanism of $\mathrm{Mn}$ incorporation, the valence state of the Mn must be strictly considered. Although some other processes have been suggested in the past, ${ }^{[25-27]}$ it is now widely accepted that $\mathrm{Mn}^{2+}$ with an ionic radius of $0.96 \AA$ (for $\mathrm{CN}=8$ ) substitutes for $\mathrm{Sr}$ on the A-site $(1.26 \AA$ and $1.44 \AA$ for coordination numbers 8 and 12 , respectivelyl[28] and $\mathrm{Mn}^{4+}(0.53 \AA)$ for $\mathrm{Ti}^{4+}(0.605 \AA)$ on the six coordinated B-site. ${ }^{[29]}$ It is possible to drive the valence state of $\mathrm{Mn}$ by the off-stoichiometry, oxygen partial pressure and temperature during heat treatment. ${ }^{[22]}$ In addition, a significant off-centering of $\mathrm{Mn}^{2+}$ from the ideal A-site position is well documented. ${ }^{[23,24,30]}$ What is not yet fully understood is how selectively we can drive the A-site and B-site incorporation. Initially, it was suggested that perfectly selective A- and B-site substitutions can be achieved, resulting in the formation of $\left(\mathrm{Sr}_{1-x} \mathrm{Mn}_{x}\right)$ $\mathrm{TiO}_{3}$ and $\mathrm{Sr}\left(\mathrm{Ti}_{1-\gamma} \mathrm{Mn}_{\gamma}\right) \mathrm{O}_{3}$ solid solutions, respectively. ${ }^{[22]}$ While B-site substitution can be performed very selectively, more accurate structural research performed using extended X-ray fine structure (EXAFS) spectroscopy has shown that A-site substitution is not selective, even when performed in a nitrogen atmosphere. ${ }^{[24]}$ Even in such conditions, Mn partially occupies the B-site. For this reason, the solid solution limit of Mn A-site substitution has never been properly determined, although attempts have been recorded. ${ }^{[31]}$ Furthermore, by ignoring the Mn misplacement, the synthesis yields a product that is not perfectly a single phase. The residual phase may exhibit some magnetic properties that can easily be mistaken for a genuine response from the matrix $\mathrm{Mn}$-doped $\mathrm{SrTiO}_{3}$. Here, we explore this behavior with particular reference to several recent publications where unusual magnetoelectric behavior of A-site Mn-doped $\mathrm{SrTiO}_{3}$ has been claimed. ${ }^{[15-17,32,33]}$

\section{Results}

To distinguish accurately between intrinsic and extrinsic sources of magnetization, an exact understanding of phase composition, substitutional mechanism and formation kinetics is crucial. This is why we started this study with a thorough analysis of $\mathrm{Mn}$ incorporation into the perovskite lattice of the $\mathrm{SrTiO}_{3}$.

During our previous studies on similar systems, ${ }^{[34]}$ we observed that, in order to improve the reaction kinetics and ensure the reaction is fully completed, high-energy milling at the stage of the oxide homogenization is essential. We performed the initial formation of $\mathrm{Mn}$-doped $\mathrm{SrTiO}_{3}$ in an air atmosphere, following the studies of Tkach et al., ${ }^{[22]}$ who suggested that the mode of $\mathrm{Mn}$ incorporation is driven by a stoichiometric deficiency on either the A- or the B-site. For nominal compositions, we assumed the following substitution mechanisms: i) for A-site substitution, $\left(\mathrm{Sr}_{1-x} \mathrm{Mn}^{2+}{ }_{x}\right) \mathrm{TiO}_{3}$; and ii) for B-site substitution, $\operatorname{Sr}\left(\mathrm{Ti}_{1-\gamma} \mathrm{Mn}^{4+}{ }_{\gamma}\right) \mathrm{O}_{3}$. The XRD patterns showed no presence of secondary phases even after long counting times. Based on the XRD patterns, we concluded that our initial assumptions above were correct; however, the phase sensitivity of the XRD analysis was limited to about 1 vol\% and might be insufficient for the small doping concentrations used in the study: this point is addressed later. Further firing of both batches was performed at a higher temperature and different oxygen partial pressures in order to obtain a better insight into the influence of the firing conditions on the Mn valence state and its distribution within the $\mathrm{SrTiO}_{3}$ lattice. Because of the limitations of the XRD technique, we decided to use Mn K-edge EXAFS and $\mathrm{X}$-ray absorption near-edge structure (XANES) spectroscopy as tools to analyze the valance state and site occupancy of the Mn.

\subsection{Mn K-Edge XANES Spectroscopy Analysis}

Mn K-edge XANES analysis was used to determine the Mn valence state in the analyzed samples. Figure 1 shows the normalized Mn XANES spectra of the samples and the reference compounds. The energy positions of the edge and pre-edge features are correlated with the valence state of the absorbing atom in the sample. ${ }^{[35-38]}$ With increasing oxidation state, the absorption edge and pre-edge features in the XANES spectrum are shifted to higher energies. We observed edge shifts of $7.4 \mathrm{eV}$ between $\mathrm{Mn}^{2+}$ and $\mathrm{Mn}^{4+}$ in the reference $\mathrm{Mn}$ compounds, $\mathrm{SrMnO}_{3}$ and $\mathrm{MnTiO}_{3}$, in agreement with previous observations. ${ }^{[24,31]}$ The energy positions of the Mn K-edge in the Mn-doped $\mathrm{SrTiO}_{3}$ samples were in the energy range between the edge positions of the two references, indicating the presence of a mixed $\mathrm{Mn}$ valence with different $\mathrm{Mn}^{2+}$ and $\mathrm{Mn}^{4+}$ ratios.

The edge shift can be determined in a straightforward way only for similar edge profiles. Different environments of the cation, most notably with different site symmetries, result in different K-edge profiles. In such cases, comparison of the edge shifts is difficult. ${ }^{[36-38]}$ Among the different approaches to determine the average valence state of the atom precisely, best results are obtained 


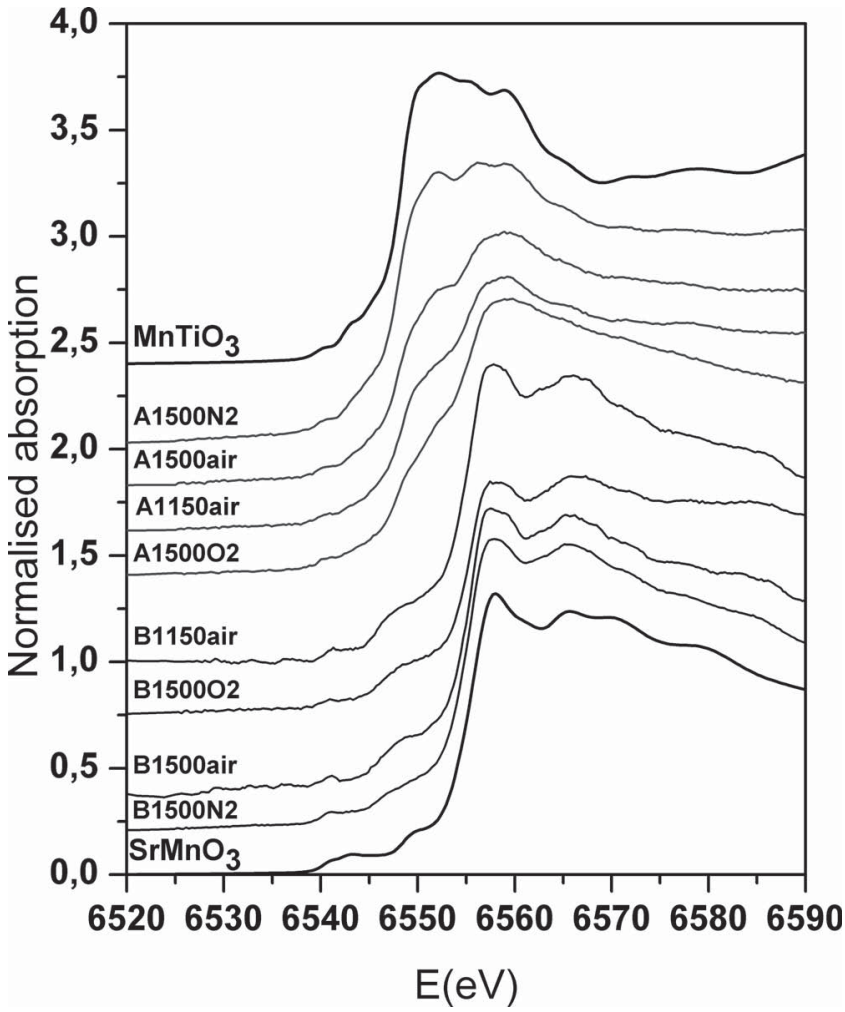

Figure 1. Mn K-edge XANES spectra of $\mathrm{Mn}$-doped $\mathrm{SrTiO}_{3}$ samples and reference manganese compounds: $\mathrm{MnTiO}_{3}$ and $\mathrm{SrMnO}_{3}$. The spectra have been shifted vertically for clarity.

by a linear combination fit with the XANES spectra of proper reference compounds, with known valence states of the element, similar symmetry, the same type of neighboring atoms in nearest coordination shells and arranged in a similar local structure. ${ }^{[36-38]}$

We applied a linear combination fit to analyze the Mn XANES spectra of Mn-doped $\mathrm{SrTiO}_{3}$ samples. The XANES spectra of all of the samples can be described very well by the linear combination of the XANES spectra of $\mathrm{MnTiO}_{3}$ and $\mathrm{SrMnO}_{3}$, as a reference for $\mathrm{Mn}^{2+}$ and $\mathrm{Mn}^{4+}$, respectively. The fit result is illustrated in Figure 2 for the A1150air sample. In this way, we were able to determine the relative ratio between $\mathrm{Mn}^{2+}$ and $\mathrm{Mn}^{4+}$ with a precision of about $2 \%$. The obtained values are listed in Table 1. As an additional test, we used the XANES spectra of the sample with the minimum amount of $\mathrm{Mn}^{4+}(\mathrm{A} 1500 \mathrm{~N} 2)$ and the sample with the maximum amount of $\mathrm{Mn}^{4+}$ (B1150air) as references to fit the spectra of the other samples. In this way, the same values of the relative ratios between $\mathrm{Mn}^{2+}$ and $\mathrm{Mn}^{4+}$ were obtained (Table 1), and the quality of the fits was even better.

\subsection{Mn K-Edge EXAFS Spectroscopy Analysis}

To identify the sites of the incorporation of the $\mathrm{Mn}^{4+}$ and $\mathrm{Mn}^{2+}$ cations in the crystal structure of $\mathrm{SrTiO}_{3}$, we measured the Mn K-edge EXAFS spectra of the B1150air sample, where the Mn was predominantly in the form of $\mathrm{Mn}^{4+}$, and the A1500N2 sample, where, predominantly, $\mathrm{Mn}^{2+}$ was present. Contributions of several consecutive shells of neighbors are visible in the Fourier

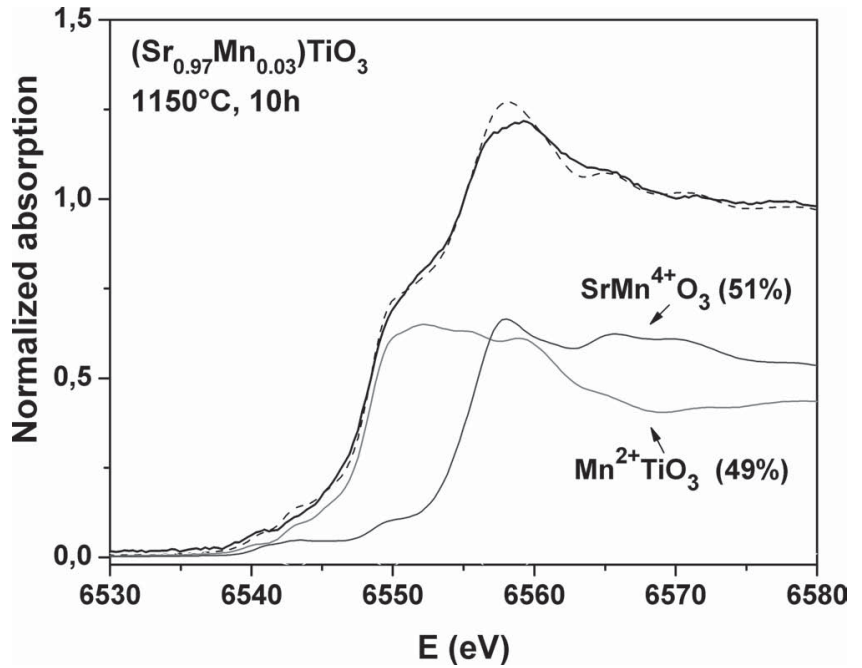

Figure 2. The $\mathrm{Mn}$ K-edge XANES spectrum of the $\left(\mathrm{Sr}_{0.97} \mathrm{Mn}_{0.03}\right) \mathrm{TiO}_{3}$ sample, which had been fired at $1150{ }^{\circ} \mathrm{C}$ in air. Black solid line: experiment; black dashed line: best-fit linear combination of XANES spectrscopy profiles of $\mathrm{MnTiO}_{3}$ (49\%) and $\mathrm{SrMnO}_{3}$ (51\%). The fit components are shown below.

transforms of the EXAFS spectra (Figure 3). In both samples, the Mn cations show well-ordered, yet significantly different local surroundings, clearly indicating that the sites of the incorporation of $\mathrm{Mn}^{4+}$ and $\mathrm{Mn}^{2+}$ in the $\mathrm{SrTiO}_{3}$ crystal structure are not the same.

In quantitative EXAFS analysis, we tested the hypothesis that $\mathrm{Mn}^{4+}$ enters the B-site, replacing $\mathrm{Ti}^{4+}$ in the structure, while $\mathrm{Mn}^{2+}$ replaces $\mathrm{Sr}^{2+}$ atoms on the A-site. Ti cations in $\mathrm{SrTiO}_{3}$ $\left(P m \overline{3} m\right.$ space group $\left.{ }^{[39]}\right)$ are octahedrally coordinated, with six oxygen atoms at $1.95 \AA$. In more distant Ti coordination shells, there are eight Sr neighbors at $3.38 \AA$, followed by six Ti and 24 $\mathrm{O}$ neighbors at $3.90 \AA$ and $4.36 \AA$, respectively. The Sr atoms, on the other hand, are surrounded by 12 oxygen atoms at 2.76 $\AA$ in the nearest coordination shell, while, in more distant coordination shells, we find eight Ti atoms at $3.38 \AA$, six Sr atoms at $3.90 \AA$ and 24 oxygen atoms at $4.77 \AA$.

For the spectrum of the B1150air sample, the EXAFS model for $\mathrm{Mn}$ on the Ti site in the $\mathrm{SrTiO}_{3}$ crystal structure ${ }^{[39]}$ was used, constructed ab initio using the FEFF6 program code ${ }^{[40]}$ from the presumed distribution of neighboring atoms around the Mn cations on the B crystallographic site. The model comprises all single and three significant multiple-scattering paths up to $4.30 \AA$. Ten parameters were varied in the model: the neighbor positions in each neighboring shell $(\Delta r)$, a separate Debye-

Table 1. Relative ratio between $\mathrm{Mn}^{2+}$ and $\mathrm{Mn}^{4+}$ in the $\mathrm{Mn}$-doped $\mathrm{SrTiO}_{3}$ samples at different synthesis conditions. The uncertainty of the obtained values is $2 \%$.

\begin{tabular}{lcc}
\hline $\begin{array}{l}\text { Temperature }\left[{ }^{\circ} \mathrm{C}\right] / \\
\text { atmosphere }\end{array}$ & $\begin{array}{r}\text { Nominal A-site doping } \\
\mathrm{Mn}^{2+} / \mathrm{Mn}^{4+}[\%]\end{array}$ & $\begin{array}{c}\text { Nominal B-site doping } \\
\mathrm{Mn}^{2+} / \mathrm{Mn}^{4+}[\%]\end{array}$ \\
\hline $1150 /$ air & $49 / 51$ & $7 / 93$ \\
$1500 / \mathrm{O}_{2}$ & $36 / 64$ & $9 / 91$ \\
$1500 /$ air & $59 / 41$ & $10 / 90$ \\
$1500 / \mathrm{N}_{2}$ & $82 / 18$ & $13 / 87$ \\
\hline
\end{tabular}




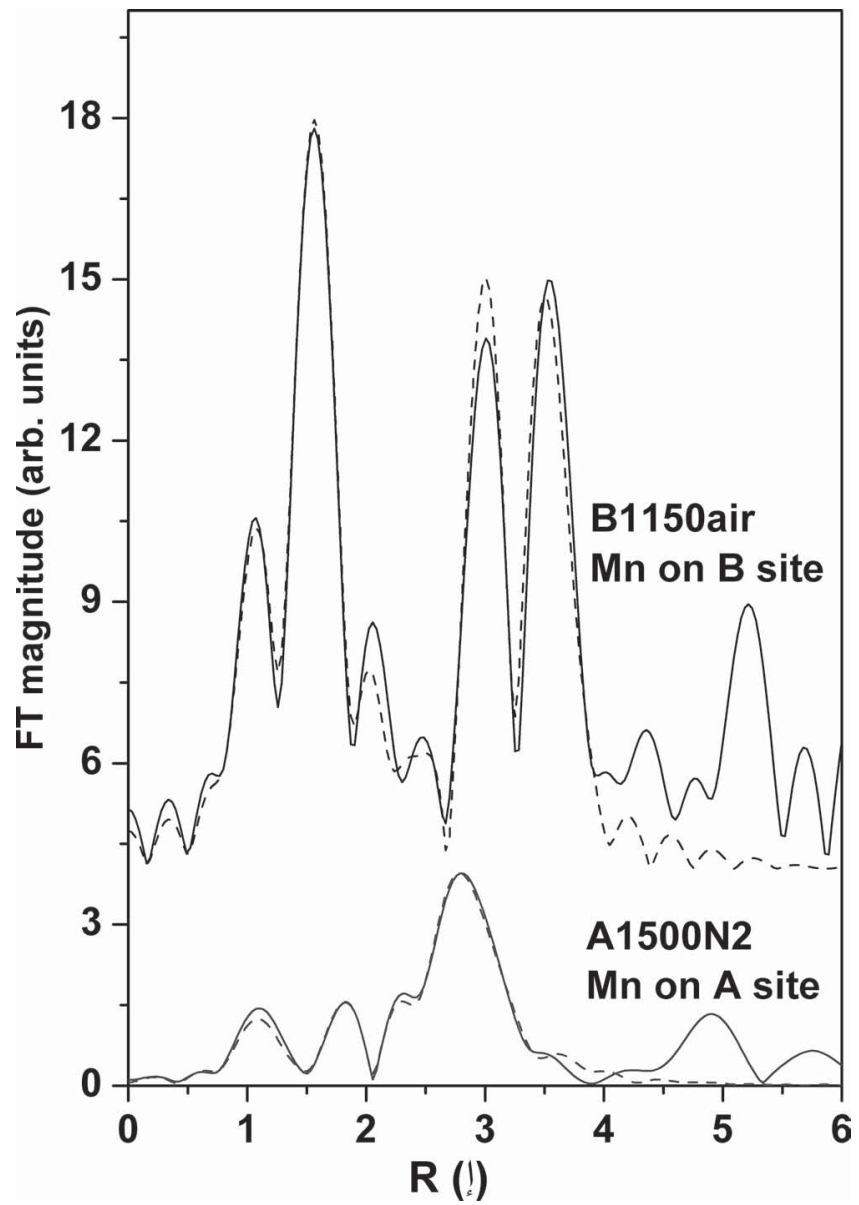

Figure 3. Fourier transform of $k^{3}$-weighted Mn EXAFS spectra of B1150air and A1500N2 samples with predominantly $\mathrm{Mn}^{4+}$ and $\mathrm{Mn}^{2+}$, respectively. Solid line: experiment; dashed line: EXAFS spectroscopy model.

Waller factor $\left(\sigma_{1}{ }^{2}\right)$ for each shell, a common shift of the energy origin $\left(\Delta E_{0}\right)$ and an amplitude reduction factor $\left(S_{0}{ }^{2}\right)$. The stoichiometry was not varied: the degeneracy of individual scattering paths was left as defined from the crystallographic data. A very good agreement between the model and the experimental spectrum was found in the $k$ range of $4 \AA^{-1}$ to $13 \AA^{-1}$ and the $R$ range from $1.2 \AA$ to $4.2 \AA$. Best-fit parameters are collected in Table 2. The quality of the fit is shown in Figure 3. The structural parameters and the value of $S_{0}{ }^{2}$ are in good agreement

Table 2. Parameters of the nearest coordination shells around $\mathrm{Mn}$ atoms in the B1150air sample: number of neighboring atoms $(N)$, distance $(R)$ and Debye-Waller factor $\left(\sigma^{2}\right)$. A best fit is obtained with the amplitude reduction factor $S_{0}{ }^{2}=0.60(3)$ and $\Delta E_{0}=2.1(9) \mathrm{eV}$. The goodness-of-fit parameter, the $R$-factor, is given in the last column.

\begin{tabular}{lcccc}
\hline Mn neighbor & $N$ & $R[\AA]]$ & $\sigma^{2}\left[\AA^{2}\right]$ & $R$-factor \\
\hline $\mathrm{O}$ & 6 & $1.90(1)$ & $0.002(1)$ & \\
$\mathrm{Sr}$ & 8 & $3.34(1)$ & $0.006(3)$ & 0.007 \\
$\mathrm{Ti}$ & 6 & $3.89(2)$ & $0.006(3)$ & \\
$\mathrm{O}$ & 24 & $4.26(2)$ & $0.011(5)$ & \\
\hline
\end{tabular}

with previous findings for $\operatorname{SrTi}_{0.98} \mathrm{Mn}_{0.02} \mathrm{O}_{3}\left[{ }^{[24]}\right.$ No distortion of the local Mn surrounding was detected, compared with that of $\mathrm{Ti}^{4+}$, except a slight relaxation of the interatomic distances. The EXAFS spectroscopy results clearly show that the $\mathrm{Mn}^{4+}$ cations substitute $\mathrm{Ti}^{4+}$ on the B crystallographic site in $\mathrm{SrTiO}_{3}$.

The EXAFS spectroscopy model for the spectrum of the A1500N2 sample is more complex. A simple model where Mn replaces $\mathrm{Sr}$ on the $\mathrm{SrTiO}_{3} \mathrm{~A}$-site ${ }^{[39]}$ is not adequate. Namely, the ionic radius of $\mathrm{Mn}^{2+}$ is significantly smaller than the radius of $\mathrm{Sr}^{2+}$. When the $\mathrm{Mn}^{2+}$ cation enters the $\mathrm{Sr}$ site, it is displaced from the central position to satisfy its bonding requirements. Levin et al. ${ }^{[24]}$ have reported that the magnitude of Mn displacements is as large as $0.77 \AA$ and is accompanied by the additional relaxation of the nearest coordination shells. Consequently, the local structure around the $\mathrm{Mn}$ is strongly distorted, compared with that around Sr.

To describe the local structure of $\mathrm{Mn}^{2+}$ on the A-sites within an $R$ range from $1.1 \AA$ to $3.8 \AA$, where the largest contributions of neighboring shells are present (Figure 3 ), we constructed an ad hoc EXAFS model, which included oxygen atoms in the nearest coordination shells at three different distances, and $\mathrm{Sr}$ and $\mathrm{Ti}$ atoms in the next coordination sphere. For each neighboring shell, two parameters were varied in the model: the neighbor positions $(\Delta r)$ and the Debye-Waller factor $\left(\sigma_{1}{ }^{2}\right)$. In addition, the common shift of the energy origin $\left(\Delta E_{0}\right)$ was allowed to vary, while the amplitude reduction factor $\left(S_{0}{ }^{2}\right.$ was fixed at the same value as in the fit for the previous sample. The coordination numbers were fixed so that the A-site stoichiometry was respected (i.e., twelve oxygen atoms in the nearest coordination shells, and eight Ti atoms in the next). Six $\mathrm{Sr}$ atoms should appear in the more distant coordination shell, but, due to the off-center position of the $\mathrm{Mn}$ in the direction of one of the Sr neighbors, we included only one $\mathrm{Sr}$ atom in the model. The other five Sr neighbors were already outside our modeling interval in $R$ space. Since XANES spectroscopy results have suggested that a small part (about 18\%) of the Mn is in the form of $\mathrm{Mn}^{4+}$, we also included an EXAFS spectroscopy model for $\mathrm{Mn}$ on the B-site in the fit (with fixed parameter values (Table 1), as obtained in the fit of the B1150air sample). The relative amounts of $\mathrm{Mn}$ at the A- and B-sites were allowed to vary.

A good fit was obtained with the parameters listed in Table 3, with an additional contribution of about 10 to $15 \%$ of $\mathrm{Mn}$ on the B-site. The structural results support the hypothesis that $\mathrm{Mn}^{2+}$

Table 3. Parameters of the nearest coordination shells around $\mathrm{Mn}$ atoms in the $A 1500 N 2$ sample: number of neighbor atoms $(N)$, distance $(R)$, and Debye - Waller factor $\left(\sigma^{2}\right)$. A best fit is obtained with the amplitude reduction factor $S_{0}^{2}=0.60$ and $\Delta E_{0}=3.5(8) \mathrm{eV}$, and $10(5) \%$ of the EXAFS spectoscopy model function for $\mathrm{Mn}$ on the $\mathrm{Ti}$ site with the structural parameters listed in Table 1. The goodness-of-fit parameter, $R$ factor, is given in the last column.

\begin{tabular}{llccc}
\hline Mn neighbor & $N$ & $R[\AA]$ & $\sigma^{2}\left[\AA^{2}\right]$ & $R$-factor \\
\hline $\mathrm{O}$ & 2 & $1.90(4)$ & $0.013(3)$ & \\
$\mathrm{O}$ & 4 & $2.18(3)$ & $0.013(3)$ & \\
$\mathrm{O}$ & 6 & $3.04(6)$ & $0.013(3)$ & 0.009 \\
$\mathrm{Sr}$ & 1 & $3.02(4)$ & $0.006(3)$ & \\
$\mathrm{Ti}$ & 4 & $3.06(3)$ & $0.011(4)$ & \\
$\mathrm{Ti}$ & 4 & $3.47(3)$ & $0.012(4)$ & \\
\hline
\end{tabular}


cations enter the A-site. However, due to differences in the $\mathrm{Mn}^{2+}$ and $\mathrm{Sr}^{2+}$ cation sizes, the local structure around the $\mathrm{Mn}$ is strongly distorted compared with that around $\mathrm{Sr}$. The $\mathrm{Mn}^{2+}$ ions shift offcenter from the A-site, and accommodate six oxygen atoms at a shorter distance (typical for $\mathrm{Mn}-\mathrm{O}$ compounds ${ }^{[37,38,41]}$ ), while the other six oxygen atoms are consequently located further away. The shell of the Ti atoms is split into two, so that four Ti neighbors are located closer and the other four are at a larger distance, as expected for the Mn off-centering in the (001) direction. The Sr neighbor appears at relatively short distance, but comparable with that reported by Levin et al. ${ }^{[2]}$

\subsection{Magnetic Measurements}

The magnetic measurements on the $\mathrm{SrTiO}_{3}$ powders treated at $1150{ }^{\circ} \mathrm{C}$ in air showed no measurable magnetic moment, apart from a very small increase in magnetization at the lowest temperatures that could have originated from magnetic impurities. The Mn-substituted samples, prepared under the same conditions, exhibited significantly higher magnetic moments with a pronounced paramagnetic tail at temperatures below $20 \mathrm{~K}$. As expected, the magnetic moment increased with the amount of $\mathrm{Mn}$. An important characteristic of these powders is that they did not show any magnetic transitions that have been previously described in the literature. In Figure 4, we show the magnetization of the A-site substituted samples after thermal treatment at $1150{ }^{\circ} \mathrm{C}$. The Mn-solubility limit for this substitutional mechanism has been estimated to be very low $(<3 \%$ according to Tkach et al. $\left.{ }^{[32]}\right)$. A very detailed study of zero-fieldcooled (ZFC) and field-cooled (FC) magnetization showed that there were no magnetic anomalies for samples up to approximately $5 \%$ of Mn. Only at $5 \%$ of Mn did the FC measurements show a small bump at around $45 \mathrm{~K}$. This temperature coincides with the ferromagnetic transition temperature of $\mathrm{Mn}_{3} \mathrm{O}_{4}{ }^{[42]}$ and indicates that the bump originates from a minor amount of residual $\mathrm{Mn}_{3} \mathrm{O}_{4}$, either segregated at the extended defect sites in

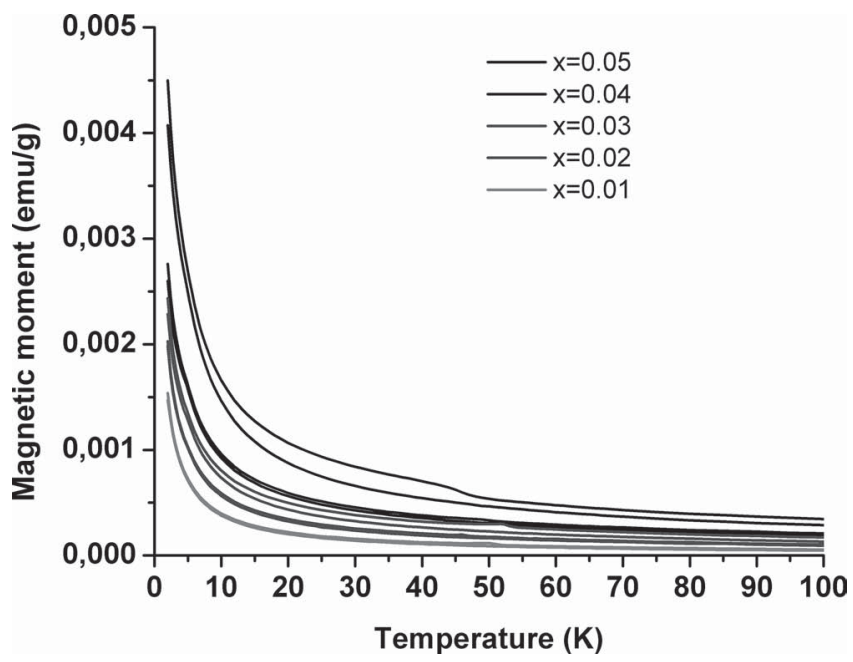

Figure 4. Magnetic moment, measured at $50 \mathrm{Oe}$, of the A-site substituted samples with nominal composition $\left(\mathrm{Sr}_{1 \cdot x} \mathrm{Mn}^{2+}{ }_{x}\right) \mathrm{TiO}_{3}$, as a function of temperature and $x$, after thermal treatment at $1150^{\circ} \mathrm{C}$. The ZFC and FC curves are essentially coincident for samples with $x<0.05$.

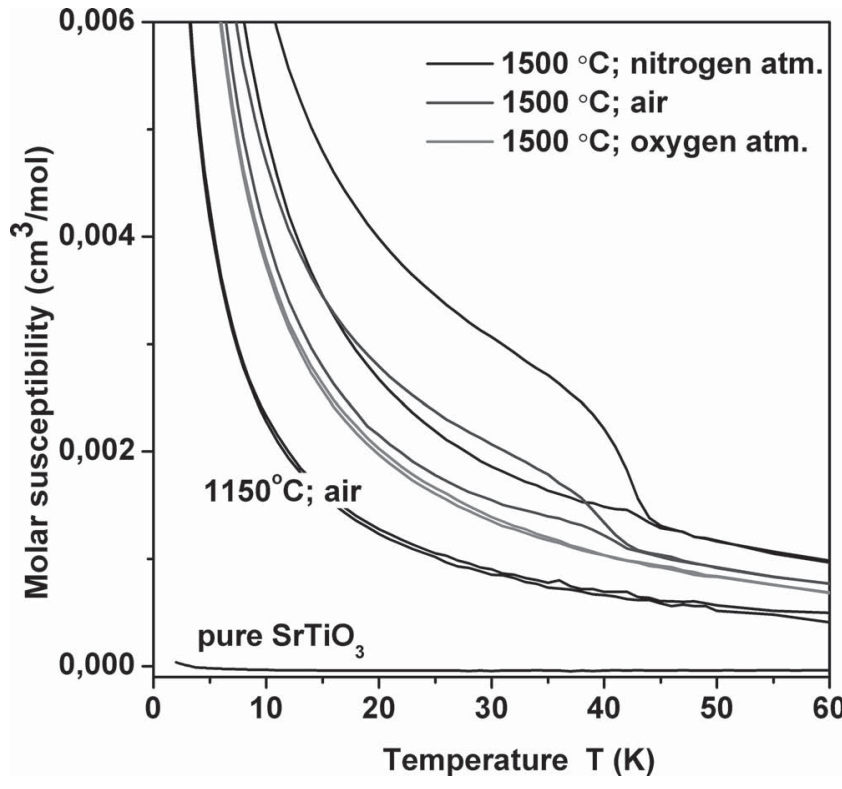

Figure 5. ZFC and FC magnetic susceptibility for the B-substituted sample with a nominal composition of $\mathrm{Sr}\left(\mathrm{Ti}_{1-\gamma} \mathrm{Mn}^{4+}{ }_{\gamma}\right) \mathrm{O}_{3}$, with $y=0.03$, as a function of temperature and heat-treatment conditions. Note that the ZFC (lower) and FC (upper) curves split below approximately $45 \mathrm{~K}$ for the samples prepared at $1500{ }^{\circ} \mathrm{C}$ in air and in a nitrogen atmosphere, due to the presence of the $\mathrm{Mn}_{3} \mathrm{O}_{4}$ impurity.

the host perovskite matrix, or precipitated as a secondary phase. Such residues are known to be present in such samples due to incomplete incorporation of Mn (either from kinetic or thermodynamic reasons). ${ }^{[34,43,44]}$ The absence of a scaling behavior between the onset temperature of the magnetic anomaly and the Mn doping level is further evidence of an extrinsic origin of the magnetic phase transition at $45 \mathrm{~K}$.

Further experiments have been performed on samples with $3 \%$ of $\mathrm{Mn}$, for which XRD and magnetic measurements showed no indication of secondary phases, and, especially, no magnetic secondary phases. In the case of the B-site doped sample treated at $1500{ }^{\circ} \mathrm{C}$ in oxygen, no magnetic anomalies were found, while one appeared at around $42 \mathrm{~K}$ for the sample treated in air. It became even more pronounced for the same sample when with treatment in a nitrogen atmosphere (Figure 5). The characteristics of the anomaly are similar to those observed for the sample with 5\% A-site substitution. Exactly the opposite is the trend for the A-site substituted samples. The same type of anomaly started to appear for the sample treated in air and became very strong for the same sample treated in oxygen (Figure 6).

\section{Discussion}

Our experiments have confirmed previous indications that the Mn-substitution mechanism can be driven by the cation deficiency of the initial $\mathrm{SrTiO}_{3}$ perovskite, the thermal treatment conditions and the oxygen partial pressure. We have shown that, at $1500^{\circ} \mathrm{C}, \mathrm{Mn}$ predominantly incorporates onto the B-site of Ti-deficient $\mathrm{SrTiO}_{3}$ in oxygen atmosphere. Predominant A-site incorporation is observed for Sr-deficient $\mathrm{SrTiO}_{3}$ treated in a nitrogen 


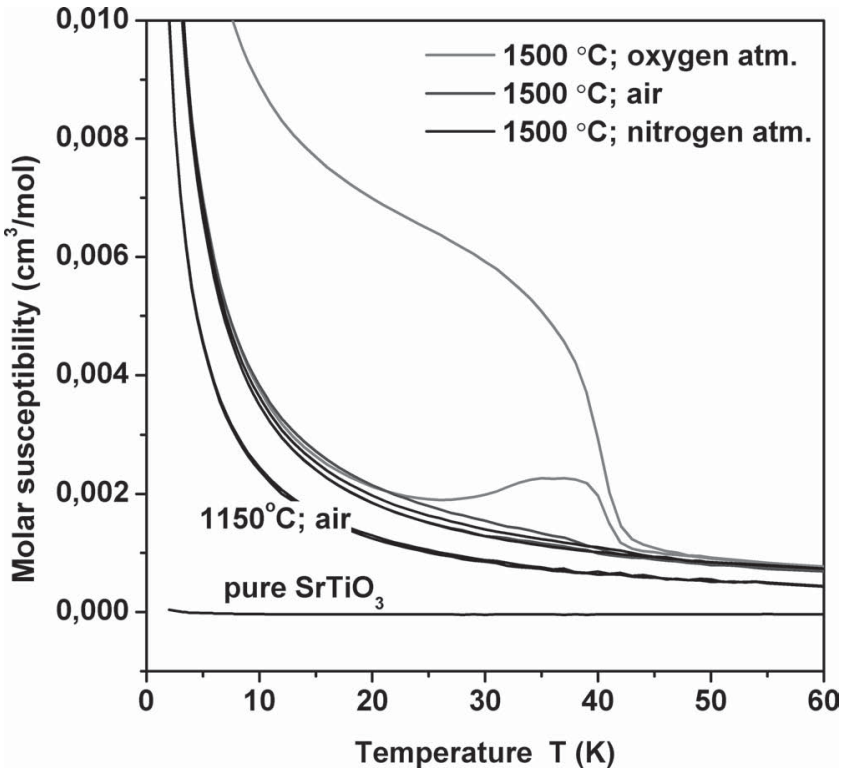

Figure 6. ZFC and FC magnetic susceptibility measured at 50 Oe for the A-substituted sample with a nominal composition of $\left(\mathrm{Sr}_{1-x} \mathrm{Mnn}_{x}^{2+}\right) \mathrm{TiO}_{3}$, with $x=0.03$, as a function of temperature and heat-treatment conditions. Note that both the ZFC (lower curve) and FC (upper curve) of the sample treated in oxygen show a magnetic anomaly at approximately $42 \mathrm{~K}$.

atmosphere. The important observation is that the samples with selective Mn incorporation do not show any of the magnetic transitions that have previously been described in the literature for $\mathrm{Mn}$-doped $\mathrm{SrTiO}_{3}$. As we have shown, the initial stoichiometric assumptions for these samples were correct, which yielded no secondary phases during the formation process, whereas the pure matrix phase gave no magnetic anomalies. Based on our experiments, we can claim that neither A-site nor B-site Mn-substitution results in intrinsic long-range magnetic order for $\mathrm{Mn}$ concentrations of $\leq 3 \%$.

This result is in disagreement with reports by Shvartsman et al. ${ }^{[16]}$ and Kleeman et al., ${ }^{[17]}$ where the authors claim that in A-site doped $\mathrm{SrTiO}_{3}$, even in the case of a low Mn concentration (2\%), the spins couple via frustrated antiferromagnetic superexchange. No evidence for such behavior has been found in our experiments although the EXAFS studies clearly showed that Mn was successfully incorporated in the $\mathrm{SrTiO}_{3}$ lattice. In our case, the conditions of the thermal treatment assured that the Mn-distribution was homogeneous enough to prevent any local Mn segregation, which would induce extrinsic spin coupling. During the heat treatment, we used a nitrogen atmosphere to drive $\mathrm{Mn}$ towards the $2+$ oxidation state, which is required for A-site substitution. The samples in the work by Shvartsman et al. ${ }^{[16]}$ and Kleeman et al. ${ }^{[17]}$ were prepared in an air atmosphere. With changes in the oxygen partial pressure, the
Mn ions alter the valence state, despite the predetermined cation deficiency of $\mathrm{SrTiO}_{3}$ (see Table 3). For the sample with $\mathrm{Mn}^{4+}$ on the B-site, a decrease in the oxygen partial pressure causes a higher proportion of reduced $\mathrm{Mn}^{2+}$. In a nitrogen atmosphere, already $13 \%$ of $\mathrm{Mn}$ is reduced. The bigger $\mathrm{Mn}^{2+}$ can no longer occupy the relatively small octahedral vacancy and is forced to move to the much bigger A-site that was previously occupied by $\mathrm{Sr}^{2+}$. This cation redistribution was confirmed with our XANES and EXAFS spectroscopy analyses. The redistribution changes the initially predetermined cation ratio, which is $\operatorname{Sr}\left(\operatorname{Ti}_{1-\gamma} \mathrm{Mn}^{4+}{ }_{\gamma}\right)$ $\mathrm{O}_{3}$, an excess of ions is removed from the perovskite matrix and can homogeneously or heterogeneously nucleate the new phases. As our magnetic measurements have shown, this process is associated with the appearance of a magnetic anomaly at around $42 \mathrm{~K}$. A higher level of the cation redistribution results in a more pronounced magnetic anomaly. Exactly the same conclusions can be drawn from the analysis of the A-site substituted $\mathrm{SrTiO}_{3}$, where the structural tolerance for the $\mathrm{Mn}$ misplacements seems to be larger. The critical level of the redistribution that is required for the appearance of the new phases depends on the thermodynamic stability of the structural vacancies in the $\mathrm{SrTiO}_{3}$, and a compensation mechanism for the misplacement. It is beyond the scope of this paper to define the mechanism of the redistribution exactly, but the characteristics of the magnetic anomaly show that it is associated with segregation of the $\mathrm{Mn}$ species and spin coupling similar to that in $\mathrm{Mn}_{3} \mathrm{O}_{4}$.

It is expected that the level of segregation required for the observed magnetic effect is too low to be detected by EXAFS and XANES spectroscopy. Therefore, we used transmission electron microscopy (TEM) to detect it. In Figure 7, we show a comparison

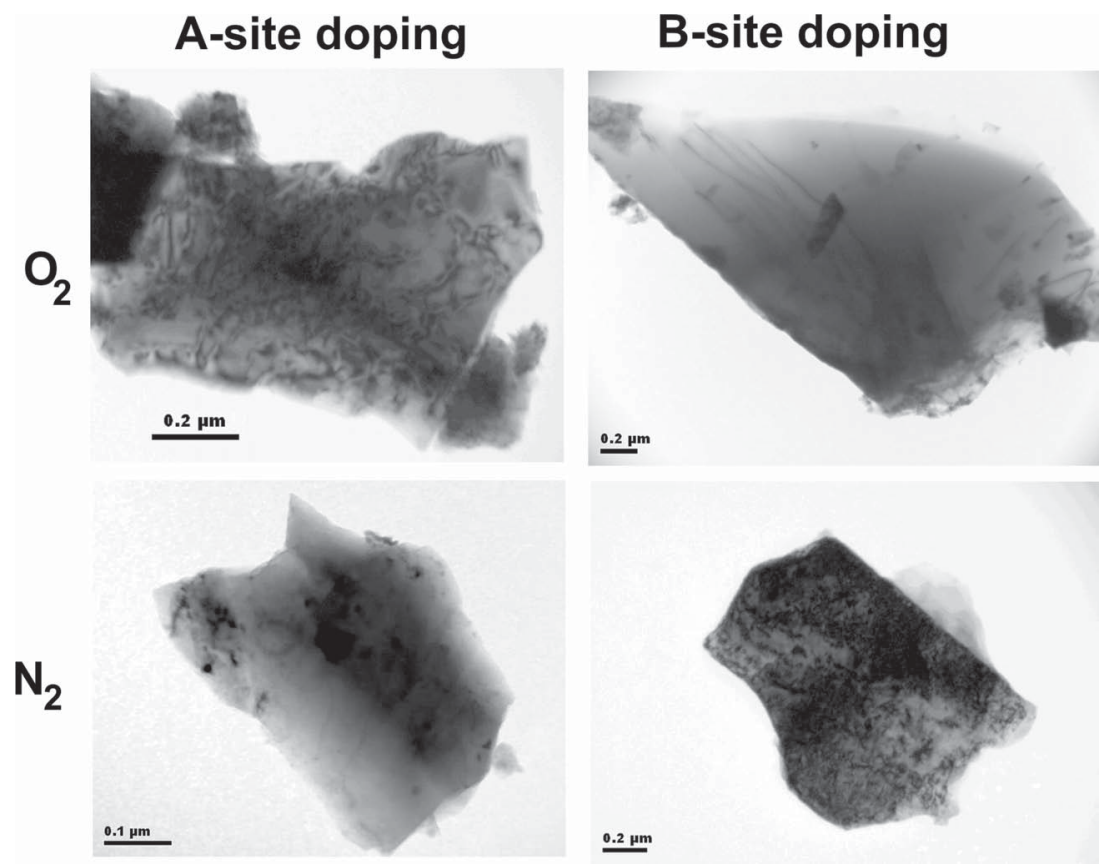

Figure 7. TEM images of $\mathrm{SrTiO}_{3}$ doped with 3 at\% $\mathrm{Mn}$ according to the nominal composition of $\left(\mathrm{Sr}_{1-x} \mathrm{Mn}_{x}^{2+}\right) \mathrm{TiO}_{3}$ and $\mathrm{Sr}\left(\mathrm{Ti}_{1-\gamma} \mathrm{Mn}_{\gamma}^{4+}\right) \mathrm{O}_{3}$ after heat treatment at $1500{ }^{\circ} \mathrm{C}$ for $10 \mathrm{~h}$. The images show a network of structural defects in the samples that show the magnetic anomaly (improperly processed) and a much more ordered structure for the samples that show no magnetic anomalies (properly processed). See Figure 5 and 6. 

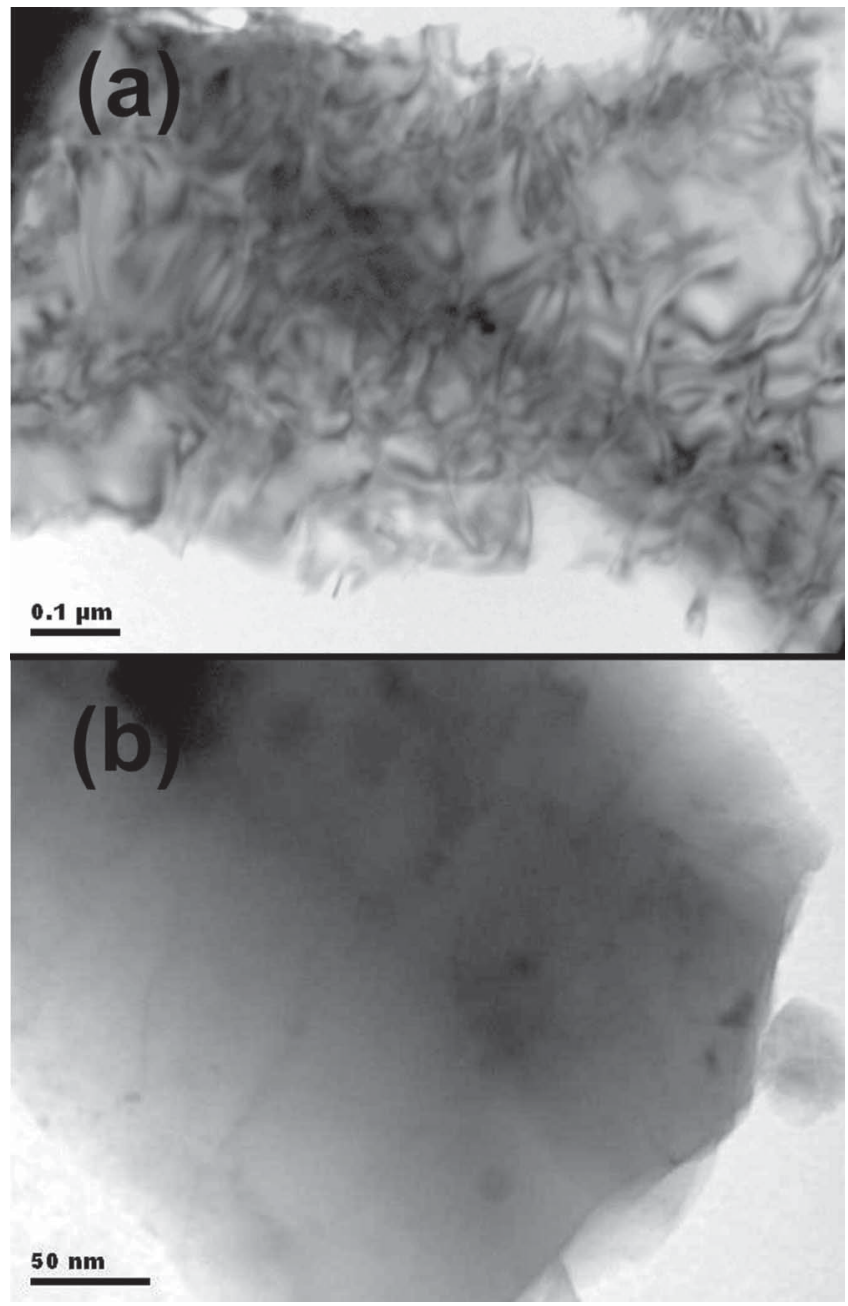

Figure 8. a,b) TEM images, close-up, of the typical microstructure of improperly (a) and properly (b) processed $\mathrm{Mn}$-doped $\mathrm{SrTiO}_{3}$ samples.

of the TEM images of the analyzed Mn-doped $\mathrm{SrTiO}_{3}$ samples, processed in oxygen and nitrogen atmospheres. The two samples, which do not show any magnetic transition, exhibit an almost defect-free, uniform microstructure. This indicates the homogeneous distribution of the dopant ions and no $\mathrm{Mn}$ segregation. In contrast, a network of microstructural features resembling dislocations is present in both the samples, which show a magnetic transition at around $42 \mathrm{~K}$ (Figure 8). The images confirm that, for these samples, the matrix was significantly strained due to changes in the valence state of the Mn ions, which could not be compensated within a single-phase field. The structure relaxed by the formation of dislocation-like structural defects, which are known low-energy sites for heterogeneous segregation/nucleation of the new phases.

We intend to analyze further the atomistic mechanisms within the observed dislocations, which are responsible for the observed spin coupling below $42 \mathrm{~K}$. However, the aim of this paper has been only to discern between the intrinsic and extrinsic sources of magnetization in $\mathrm{Mn}$-doped $\mathrm{SrTiO}_{3}$. Our experimental results have made it very clear that the origin of the magnetization in $\mathrm{Mn}$-doped $\mathrm{SrTiO}_{3}$ is not intrinsic, but rather extrinsic. Properly prepared and processed Mn-doped $\mathrm{SrTiO}_{3}$ samples do not exhibit long-range magnetic order or a spin-glass state. Improper material processing causes initially unpredicted changes in the valence state of the $\mathrm{Mn}$ and results in the formation of structural defects and irregularities associated with the segregation and nucleation of magnetic species. Only such samples exhibit a magnetic phase transition at around $42 \mathrm{~K}$, which is of extrinsic origin. This transition temperature closely corresponds to the transition temperature in the tetragonal $\mathrm{Mn}_{3} \mathrm{O}_{4}$ spinel phase, which suggests that the spincoupling mechanism of segregated $\mathrm{Mn}$ ions is also similar. We suggest that the partial solubility of Ti ions in the $\mathrm{Mn}_{3} \mathrm{O}_{4}$ host may further decrease the temperature of the magnetic anomaly due to a magnetic dilution effect. It is worth mentioning that the magnetoelectric behavior was confirmed for $\mathrm{Mn}_{3} \mathrm{O}_{4},{ }^{[42]}$ which might be the source of a similar effect observed in poorly processed Mn-doped $\mathrm{SrTiO}_{3}$.

\section{Conclusions}

The influence of the processing parameters and off-stoichiometry on the incorporation of $\mathrm{Mn}$ in the $\mathrm{SrTiO}_{3}$ crystal structure has been carefully analyzed. We have shown that, under proper conditions, $3 \%$ of $\mathrm{Mn}$ can fully incorporate into $\mathrm{SrTiO}_{3}$, predominantly on the A-site as $\mathrm{Mn}^{2+}$ or the B-site as $\mathrm{Mn}^{4+}$. The conditions for such incorporation are given in this paper. Such samples do not exhibit any evidence of long-range magnetic interactions or a spin-glass, down to as low as $5 \mathrm{~K}$. A magnetic anomaly was only observed for the samples in which Mn was misplaced, due to changes in the processing conditions and the valence state of the $\mathrm{Mn}$.

Our findings conflict with claims, reported in several papers, that $\mathrm{Mn}$-doped $\mathrm{SrTiO}_{3}$ exhibits intrinsic spin coupling via frustrated antiferromagnetic superexchange, a low-temperature spin glass state and magnetoelectric coupling. ${ }^{[16,17]}$ We have noticed no indication for the existence of these phenomena in properly processed $\mathrm{Mn}$-doped $\mathrm{SrTiO}_{3}$. The chemistry of these materials is much more complex than previously assumed and should be carefully controlled and precisely understood before the magnetic properties can be interpreted reliably. The extrinsic reasons for the observed magnetic effects might not be traceable by standard analytical techniques and, therefore, easily mistaken for the intrinsic signal. Appealing and somewhat exotic physical models are proposed for model systems. Unfortunately, they are based on signals measured for samples that are, from the (micro)structural point of view, fundamentally different from model systems.

\section{Experimental Section}

The starting powders of $\mathrm{SrCO}_{3}(99.9 \%), \mathrm{TiO}_{2}(99.9 \%)$ and $\mathrm{MnO}_{2}(99.9 \%)$ were mixed in stoichiometric ratios corresponding to the substitutional formulae $\left(\mathrm{Sr}_{1-x} \mathrm{Mn}^{2+}{ }_{x}\right) \mathrm{TiO}_{3}$ and $\mathrm{Sr}\left(\mathrm{Ti}_{1-\gamma} \mathrm{Mn}^{4+}{ }_{\gamma}\right) \mathrm{O}_{3}$, with $x$ and $y$ between 0.00 and 0.05 . The starting powders were homogenized by highenergy milling in a planetary mill (Struers) at $300 \mathrm{rpm}$ for $0.5 \mathrm{~h}$ using yttria-stabilized zirconia balls and a jar. Firing of the two batches was performed in air at $1150^{\circ} \mathrm{C}$ for $10 \mathrm{~h}$. After the initial firing, the powders were milled in a planetary mill and the batches were divided into 
several smaller batches. They were fired at $1500{ }^{\circ} \mathrm{C}$ for $10 \mathrm{~h}$ in different atmospheres: oxygen, air or nitrogen atmospheres. The annotation for the $x=0.03$ samples is the following: substitution site/temperature/ atmosphere; for instance, the annotation A1500air refers to the sample with $3 \% \mathrm{Mn}$ at the $\mathrm{A}$-site fired at $1500{ }^{\circ} \mathrm{C}$ in air.

The structural and phase analyses were carried out using a powder $\mathrm{X}$-ray diffractometer (Philips X'pert Pro), with $\mathrm{Cu} \mathrm{K}_{\alpha}$ radiation and a highthroughput detector (X'Celerator, PANalytical). Magnetic measurements were performed in the 2-100 $\mathrm{K}$ temperature range using commercial Quantum Design Magnetic Property Measurements system equipped with a superconducting quantum interference device (SQUID). The magnetic moments were measured upon heating for both zero-fieldcooled (ZFC) and field-cooled (FC) conditions. The microstructure imaging was performed using a JEOL 2000FX transmission electron microscope (TEM) operating at an accelerating voltage of $200 \mathrm{kV}$. The specimens were prepared on a copper grid from a suspension of $\mathrm{Mn}$ :STO powder in methanol.

The X-ray-absorption spectra of the $\mathrm{Mn}$-doped $\mathrm{SrTiO}_{3}$ samples and the reference manganese compounds, $\mathrm{SrMnO}_{3}$ and $\mathrm{MnTiO}_{3}$, with known $\mathrm{Mn}$ valence states and structures, were measured in the $\mathrm{Mn} \mathrm{K}$-edge energy range at room temperature in transmission- and fluorescence-detection mode on the $C$ beamline at Hamburger Synchrotronstrahlungslabor (HASYLAB) at the Deutsches Elektronen-Synchrotron (DESY) and at the X-ray absorption fine structure (XAFS) beamline at the Elettra Laboratory. At both beamlines, a Si (111) double-crystal monochromator was used at $6.5 \mathrm{keV}$, with a resolution of about $0.8 \mathrm{eV}$. Higher-order harmonics were effectively eliminated by detuning of the monochromator crystals to $60 \%$ of the rocking-curve maximum. The intensity of the monochromatic $\mathrm{X}$-ray beam was measured using three consecutive ionization detectors. On the $C$ beamline, $10 \mathrm{~cm}$-long ionization chambers were used, respectively filled with 960 mbar $\mathrm{N}_{2}, 315 \mathrm{mbar}$ Ar and 700 mbar Ar. On the XAFS beamline, the $30 \mathrm{~cm}$-long ionization chambers were filled with the following gas mixtures: $580 \mathrm{mbar}_{2}$ and $1420 \mathrm{mbar} \mathrm{He} ; 1000 \mathrm{mbar}$ $\mathrm{N}_{2}, 90 \mathrm{mbar} \mathrm{Ar}$ and $910 \mathrm{mbar} \mathrm{He} ; 350 \mathrm{mbar} \mathrm{Ar}, 1000 \mathrm{mbar}^{\mathrm{N}}$ and 650 mbar He.

The samples were prepared as self-supporting pellets that were pressed from a homogenous mixture of the sample and boron nitride powder, with a total absorption thickness of about 2.5, above the $\mathrm{Mn}$ K-edge. The pellets were placed between the first pair of ionization detectors. For the low-Mn content samples, where a Mn K-edge jump of only about 0.05 was obtained in transmission detection mode, fluorescence detection mode was used (in parallel with the transmission), exploiting the seven-pixel $\mathrm{Si}(\mathrm{Li})$ fluorescence detector at the $\mathrm{C}$ station of HASYLAB and the silicon drift detector (SDD) on the XAFS beamline at Elettra. In this case, the absorption spectra were obtained as the ratio of the fluorescence detector signal and the signal of the incident photon beam from the first ionization chamber.

In the XANES-spectroscopy region, equidistant energy steps of $0.25 \mathrm{eV}$ were used, while for the EXAFS spectroscopy region, equidistant $k$ steps of $0.03 \AA^{-1}$ were adopted, with an integration time of $4 \mathrm{~s}$ per step. Three repetitions were collected for each sample and superimposed to improve the signal-to-noise ratio. In all of the experiments, the exact energy calibration was established by simultaneous absorption measurements on a $5 \mu \mathrm{m}$-thick $\mathrm{Mn}$ metal foil placed between the second and the third ionization chambers. The absolute energy reproducibility of the measured spectra was $\pm 0.03 \mathrm{eV}$. The analysis of the XANES and EXAFS spectra was performed using the IFEFFIT software package. ${ }^{[45]}$

\section{Acknowledgements}

This work was supported by the Slovenian Research Agency under grant no. 12-2007-1540, by DESY and the European Community's Seventh Framework Programme (FP7/2007-2013) ELISA (European Light Sources Activities) under grant agreement no. 226716, and by the Engineering and Physical Sciences Research Council (EPSRC), UK. Access to the synchrotron-radiation facilities at HASYLAB (beamline C) and Elettra (beamline XAFS) is acknowledged. The authors thank Adam
Webb, Roman Chernikov and Edmund Welter of HASYLAB, and Giuliana Aquilanti and Luca Olivi of Elettra, for their support and expert advice on beamline operation. T.K. was supported by a Grant in Aid for Scientific Research C 21560025 from MEXT, Japan.

Received: October 14, 2011

Revised: December 11, 2011 Published online:

[1] P. Sharma, A. Gupta, K. V. Rao, F. J. Owens, R. Sharma, R. Ahuja, J. M. O. Guillen, B. Johansson, G. A. Gehring, Nat. Mater. 2003, 2, 673.

[2] G. Peleckis, X. L. Wang, S. X. Dou, Appl. Phys. Lett. 2006, 88, 132507.

[3] S. B. Ogale, R. J. Choudhary, J. P. Buban, S. E. Lofland, S. R. Shinde, S. N. Kale, V. N. Kulkarni, J. Higgins, C. Lanci, J. R. Simpson, N. D. Browning, S. Das Sarma, H. D. Drew, R. L. Greene, T. Venkatesan, Phys. Rev. Lett. 2003, 91, 077205.

[4] Y. Matsumoto, M. Murakami, T. Shono, T. Hasegawa, T. Fukumura, M. Kawasaki, P. Ahmet, T. Chikyow, S. Koshihara, H. Koinuma, Science 2001, 291, 854.

[5] D. P. Norton, N. A. Theodoropoulou, A. F. Hebard, J. D. Budai, L. A. Boatner, S. J. Pearton, R. G. Wilson, Electrochem. Solid-State Lett. 2003, 6, G19.

[6] J. M. D. Coey, Curr. Opin. Solid State Mater. Sci. 2006, 10, 83.

[7] J. M. D. Coey, S. A. Chambers, MRS Bull. 2008, 33, 1053.

[8] M. Vopsaroiu, M. G. Cain, P. D. Woolliams, P. M. Weaver, M. Stewart, C. D. Wright, Y. Tran, J. Appl. Phys. 2011, 109, 066101.

[9] K. Yatesin Nanomagnetism and Spintronics: Fabrication, Materials, Characterization and Applications, (Eds: F. Nasirpouri, A. Nogaret), World Scientific Publishing Co. Pte. Ltd., Singapore 2011, Ch. 10.

[10] M. A. Garcia, E. F. Pinel, J. de la Venta, A. Quesada, V. Bouzas, J. F. Fernandez, J. J. Romero, M. S. Martin-Gonzalez, J. L. Costa-Kramer, J. Appl. Phys. 2009, 105, 013925.

[11] J. M. D. Coey, J. T. Mlack, M. Venkatesan, P. Stamenov, IEEE Trans. Magn. 2010, 46, 2501.

[12] M. A. Garcia, F. Jimenez-Villacorta, A. Quesada, J. de la Venta, N. Carmona, I. Lorite, J. Llopis, J. F. Fernandez, J. Appl. Phys. 2010, 107,043906

[13] J. M. D. Coey, K. Wongsaprom, J. Alaria, M. Venkatesan, J. Phys. D: Appl. Phys. 2008, 41, 134012.

[14] M. S. Martin-Gonzalez, M. A. Garcia, I. Lorite, J. L. Costa-Kramer, F. Rubio-Marcos, N. Carmona, J. F. Fernandez, J. Electrochem. Soc. 2010, 157, E31.

[15] R. O. Kuzian, V. V. Laguta, A. M. Daré, I. V. Kondakova, M. Marysko, L. Raymond, E. P. Garmash, V. N. Pavlikov, A. Tkach, P. M. Vilarinho, R. Hayn, EPL 2010, 92, 17007.

[16] V. V. Shvartsman, S. Bedanta, P. Borisov, W. Kleemann, A. Tkach, P. M. Vilarinho, Phys. Rev. Lett. 2008, 101, 165704.

[17] W. Kleemann, V. V. Shvartsman, S. Bedanta, P. Borisov, A. Tkach, P. M. Vilarinho, J. Phys.: Condens. Matter 2008, 20, 434216.

[18] C. B. Azzoni, M. C. Mozzati, A. Paleari, V. Massarotti, M. Bini, D. Capsoni, Solid State Commun. 2000, 114, 617.

[19] A. A. Belik, Y. Matsushita, Y. Katsuya, M. Tanaka, T. Kolodiazhnyi, M. Isobe, E. Takayama-Muromachi, Phys. Rev. B: Condens. Matter 2011, 84, 094438

[20] J. S. Lee, Z. G. Khim, Y. D. Park, D. P. Norton, N. A. Theodoropoulou, A. F. Hebard, J. D. Budai, L. A. Boatner, S. J. Pearton, R. G. Wilson, Solid State Electron. 2003, 47, 2225.

[21] P. Galinetto, A. Casiraghi, M. C. Mozzati, C. B. Azzoni, D. Norton, L. A. Boatner, V. Trepakov, Ferroelectrics 2008, 368, 120.

[22] A. Tkach, P. Vilarinho, A. Kholkin, Acta Mater. 2006, 54, 5385.

[23] I. V. Kondakova, R. O. Kuzian, L. Raymond, R. Hayn, V. V. Laguta, Phys. Rev. B: Condens. Matter 2009, 79, 134117. 
[24] I. Levin, V. Krayzman, J. C. Woicik, A. Tkach, P. M. Vilarinho, Appl. Phys. Lett. 2010, 96, 052904.

[25] R. A. Serway, W. Berlinger, K. A. Muller, R. W. Collins, Phys. Rev. B: Condens. Matter 1977, 16, 4761.

[26] Y. Iwazaki, T. Suzuki, H. Kishi, S. Tsuneyuki, IEEE Int. Symp. Appl. Ferroelectr. 2007, 249.

[27] M. Savinov, V. A. Trepakov, P. P. Syrnikov, V. Zelezny, J. Pokorny, A. Dejneka, L. Jastrabik, P. Galinetto, J. Phys.: Condens. Matter 2008, 20, 095221

[28] Here we only report the ionic size of $\mathrm{Mn}^{2+}$ for co-ordination number 8 for two reasons: i) the ionic size for co-ordination number 12 is, to our knowledge, not available; and ii) $\mathrm{Mn}^{2+}$ ions displace from their ideal perovskite A-position so much that the lower co-ordination is justified.

[29] A. Tkach, P. M. Vilarinho, A. L. Kholkin, I. M. Reaney, J. Pokorny, I. Petzelt, Chem. Mater. 2007, 19, 6471.

[30] R. D. Shannon, C. T. Prewitt, J. Inorg. Nucl. Chem. 1970, 32, 1427.

[31] A. I. Lebedev, I. A. Sluchinskaya, A. Erko, V. F. Kozlovskii, JETP Lett. 2009, 89, 457.

[32] A. Tkach, P. M. Vilarinho, A. L. Kholkin, Acta Mater. 2005, 53, 5061.

[33] W. Kleemann, P. Borisov, V. V. Shvartsman, S. Bedanta, A. Tkach, P. M. Vilarinho, J. Magn. Magn. Mater. 2009, 321, 1785.

[34] M. Valant, T. Kolodiazhnyi, A. K. Axelsson, G. S. Babu, N. M. Alford, Chem. Mater. 2010, 22, 1952.
[35] J. Wong, F. W. Lytle, R. P. Messmer, D. H. Maylotte, Phys. Rev. B: Condens. Matter 1984, 30, 5596.

[36] I. Arčon, J. Kolar, A. Kodre, D. Hanžel, M. Strli, X-ray Spectrom 2007, 36, 199

[37] R. Dominko, I. Arcon, A. Kodre, D. Hanzel, M. Gaberscek, J. Power Sources 2009, 189, 51.

[38] R. Dominko, C. Sirisopanaporn, C. Masquelier, D. Hanzel, I. Arcon, M. Gaberscek, J. Electrochem. Soc. 2010, 157, A1309.

[39] Y. A. Abramov, V. G. Tsirelson, V. E. Zavodnik, S. A. Ivanov, I. D. Brown, Acta Crystallogr. B 1995, 51, 942.

[40] J. J. Rehr, R. C. Albers, S. I. Zabinsky, Phys. Rev. Lett. 1992, 69, 3397.

[41] N. Novak Tusar, A. Ristic, G. Mali, M. Mazaj, D. Arcon, I. Arcon, V. Kaucic, N. Zabukovec Logar, Chem. Eur. J. 2010, 16, 5783.

[42] R. Tackett, G. Lawes, B. C. Melot, M. Grossman, E. S. Toberer, R. Seshadri, Phys. Rev. B: Condens. Matter 2007, 76, 024409.

[43] D. Berardan, E. Guilmeau, D. Pelloquin, J. Magn. Magn. Mater. 2008, 320, 983.

[44] D. C. Kundaliya, S. B. Ogale, S. E. Lofland, S. Dhar, C. J. Metting, S. R. Shinde, Z. Ma, B. Varughese, K. V. Ramanujachary, L. Salamanca-Riba, T. Venkatesan, Nat. Mater. 2004, 3, 709.

[45] B. Ravel, M. Newville, J. Synchrotron Radiation 2005, 12, 537. 